Pacific Journal of Mathematics

INVARIANT SUBSPACE LATTICES AND COMPACT
OPERATORS 


\title{
INVARIANT SUBSPACE LATTICES AND COMPACT OPERATORS
}

\section{Cecelia Laurie}

\begin{abstract}
A general question is what can the invariant subspaces of a compact operator look like. To obtain information about this, we examine certain lattices of subspaces of a separable Hilbert space with the intent of determinig whether such a lattice could be left invariant by a compact operator.
\end{abstract}

Identify a lattice of subspaces with the lattice of projections onto those subspaces and consider only those lattices which are commutative and closed in the strong operator topology. The subclass of lattices examined are those which are multiplicity free (in the sense that the algebra generated by the projections is a maximal abelian self-adjoint algebra) and are generated as a lattice by a finite number of (mutually commuting) totally ordered sublattices. It is found that, although not all such lattices are left invariant by a compact operator, if the generating sublattices satisfy a natural independence condition, then there will be a compact, in fact Hilbert-Schmidt, operator that will leave the lattice invariant.

1. Introduction. Let $\mathscr{H}$ be a separable Hilbert space. If $P$ and $Q$ are two projections on $\mathscr{H}$ then their meet, $P \wedge Q$, is the projection onto the intersection of their ranges and their join, $P \vee Q$, is the projection onto the subspace union of their ranges. These operations along with the partial ordering of inclusion of ranges enables us to talk of lattices of projections. The term subspace lattice will refer to a lattice of projections on $\mathscr{H}$ which is closed in the strong operator topology and contains 0 , the zero projection, and $I$, the identity projection. We restrict our attention to commutative subspace, lattices. We will call a totally ordered subspace lattice a chain.

Ringrose has shown that, given any chain on $\mathscr{H}$, there is a compact (in fact rank 1) operator leaving the chain invariant ([5], Lemma 3.3). The question arises of what other possible (commutative) subspace lattices are left invariant by some compact operator. A natural class of subspace lattices to consider are those generated by a finite number of mutually commuting chains.

We first note that not all such lattices can be left invariant by a compact operator. Let $\mathscr{C}$ be a chain of projections on $\mathscr{H}$ and consider the subspace lattice $\mathscr{B}$ generated by the two chains $\mathscr{C}$ 
and $\mathscr{C}^{\perp}=\{I-P: P \in \mathscr{C}\}$. (Any subspace lattice $\mathscr{H}$ which is closed under taking orthogonal complements can be decomposed in this manner. See [1], p. 484.) Suppose further that $\mathscr{C}^{\prime \prime}$, the von Neumann algebra generated by $\mathscr{C}$, is nonatomic, i.e., that every nonzero projection in $\mathscr{C}^{\prime \prime}$ has a nontrivial subprojection in $\mathscr{C}^{\prime \prime}$. Then $\mathscr{B}$ cannot be left invariant by a compact operator.

(To see this, first note that if an operator $K$ leaves $\mathscr{B}$ invariant, then each projection in $\mathscr{B}$ corresponds to a reducing subspace for $K$. Since $\mathscr{C}^{\prime \prime}$ is nonatomic, one can regard $\mathscr{C}$ as a "continuous" spread of projections from 0 to $I$; more specifically there is a one-one (order preserving) correspondence between $\mathscr{C}$ and the unit interval $[0,1]$. (See Prop. 3.2.) It is also known that every compact operator on $\mathscr{H}$ has a nontrivial irreducible subspace. Now suppose that $K$ is compact and leaves $\mathscr{B}$ invariant. Letting $Q$ be the projection onto a nontrivial irreducible subspace for $K$, we have that $P \wedge Q$ reduces $K$ for every $P \in \mathscr{C}$ and hence that $P \wedge Q=0$ or $Q$. Since $0 \in \mathscr{C}$ and $I \in \mathscr{C}$, this would imply that for some $t_{0}$ in $[0,1]$ the projection $P_{0}$ in $\mathscr{C}$ corresponding to $t_{0}$ would satisfy $P_{0} \wedge Q=0$ and $P_{0} \wedge Q=Q$, a contradiction.)

Hence we wish to examine further the structure of subspace lattices generated by a finite number of mutually commuting chains. This is facilitated by restricting our attention to multiplicity free lattices. (A commutative subspace lattice $\mathscr{L}$ is multiplicity free if the von Neumann algebra generated by $\mathscr{L}$ is a maximal abelian self adjoint algebra.) In $\S 3$, we show that a multiplicity free lattice $\mathscr{L}$ generated (as a subspace lattice) by $n$ chains is unitarily equivalent to a certain lattice of projections in the multiplication algebra of $L^{2}\left([0,1]^{n}, m\right)$ for some finite Borel measure $m$. Call this lattice $\hat{\mathscr{L}}$. In $\S 4$,we introduce the notion of independence of chains, i.e., we say $\mathscr{C}_{1}, \cdots, \mathscr{C}_{n}$ are independent if for every $E_{i}, F_{i} \in \mathscr{C}_{i}$, we have that $E_{1} \wedge \cdots \wedge E_{n} \leqq F_{1} \vee \cdots \vee F_{n}$ implies that $E_{j} \leqq F_{j}$ for some $j$. The chains generating $\mathscr{L}$ being independent induces a property of the "representation" measure $m$ from which we can deduce the existence of a Hilbert-Schmidt operator on $L^{2}\left([0,1]^{n}, m\right)$ which leaves $\hat{\mathscr{L}}$ invariant. (See $\S 5$.) Because $\mathscr{L}$ and $\hat{\mathscr{L}}$ are unitarily equivalent, it follows that there is a Hilbert-Schmidt operator on $\mathscr{H}$ which leaves $\mathscr{L}$ invariant.

The results in this paper are part of the author's doctoral dissertation at the University of California, Berkeley and she wishes to express her appreciation to her adviser, William Arveson, for his guidance, assistance, and encouragement.

2. Terminology and notation. Throughout this paper, $\mathscr{C}$ 
will denote a separable Hilbert space. We collect here some standard notation and terms that will be used.

If $\mathscr{Z}$ is a self-adjoint collection of bounded operators in $\mathscr{L}(\mathscr{H})$, then $\mathscr{Z}^{\prime \prime}$ is the double commutant which equals the weakly (strongly) closed algebra generated by $\mathscr{z}$.

A m.a.s.a. is a maximal abelian self-adjoint algebra of operators.

An algebra of bounded operators is called nonatomic if there are no minimal projections for the algebra. A minimal projection for an algebra is a projection $P$ such that every subprojection of $P$ in the algebra is either 0 or $P$.

If $X$ is a set with a specified $\sigma$-field ( $\sigma$-algebra) of subsets, a spectral measure on $X$ is a function $P$ whose domain is the $\sigma$-field of subsets and whose values are projections on $\mathscr{H}$ such that $P(X)=I$ and such that $P\left(\bigcup_{n} M_{n}\right)=\sum_{n} P\left(M_{n}\right)$ whenever $\left\{M_{n}\right\}$ is a disjoint sequence of subsets in the $\sigma$-field.

The compact spectral measure associated with a self-adjoint operator $A$ is the unique spectral measure $P$ defined on Borel sets of the real line having compact support such that $A=\int t d P(t)$ where this notation means that $(A x, y)=\int t d \mu_{x y}(t)$ for $\mu_{x y}(M)=(P(M) x, y)$. (See [3], §43.)

A spectral projection of $A$ is a projection of the form $P(M)$ for $M$ a Borel set where $P$ is the spectral measure associated with $A$.

Most of the following terminology, used throughout the paper, is that introduced by Arveson [1].

A subspace lattice $\mathscr{L}$ is a lattice of projections on $\mathscr{H}$ which contains 0 and $I$ (the identity), and is closed in the strong operator topology. Throughout the rest of the paper the term lattice (of projections) will mean subspace lattice.

A chain is a totally ordered subspace lattice.

A subspace lattice $\mathscr{L}$ is said to have finite width if there is a finite set of chains $\mathscr{C}_{1}, \cdots, \mathscr{C}_{n}$ such that $\mathscr{L}$ is the subspace lattice generated by $\mathscr{C}_{1}, \cdots, \mathscr{C}_{n}$. We denote this by $\mathscr{L}=\mathscr{C}_{1} \mathrm{~V} \cdots \mathrm{V} \mathscr{C}_{n}$. We will call a subspace lattice $\mathscr{L}$ multiplicity free if $\mathscr{L}^{\prime \prime}$ is a m.a.s.a.

A partial ordering $\leqq$ on a set $X$ is an order relation that is reflexive and transitive, i.e., $x \leqq x$ and if $x \leqq y$ and $y \leqq z$, then $x \leqq z$.

The partially ordered topological space $(X, \leqq)$ is said to be standard if

(i) $X$ is standard in the sense that it is Borel isomorphic to a Borel subset of a separable complete metric space in the relative Borel structure.

(ii) There exists a sequence $f_{1}, f_{2}, \cdots$ of real valued Borel functions on $X$ such that, for all $x$ and $y$ in $X, x \leqq y$ iff $f_{n}(x) \leqq f_{n}(y)$ 
for $n \geqq 1$. This is equivalent to the existence of a sequence of Borel sets $E_{1}, \cdots, E_{n}, \cdots$ such that $x \leqq y$ iff $\chi_{E_{n}}(x) \leqq \chi_{E_{n}}(y)$ for every $n$.

The graph $G$ of $(X, \leqq)$ is defined by $G=\{(x, y) \in X \times X: y \leqq x\}$.

A standard partially ordered measure space $(X, \leqq, m)$ is a standard partially ordered topological space with a $\sigma$-finite regular Borel measure $m$.

Let $(X, \leqq, m)$ be a standard partially ordered measure space. A Borel set $E$ is said to be increasing if for $x \in E, x \leqq y$ implies $y \in E$. Define $\mathscr{L}(X, \leqq, m)$ as $\left\{P_{E}: E\right.$ increasing $\}$ where $P_{E}$ is the projection on $L^{2}(X, m)$ formed by multiplication by $\chi_{E} \cdot \quad \mathscr{L}(X, \leqq, m)$ forms a subspace lattice.

3. A representation theorem for multiplicity free finite width lattices. If $\mathscr{L}$ is a commutative lattice, then $\mathscr{L}$ can be represented as a lattice of projections in the multiplication algebra of some $L^{2}$ space, i.e., Arveson has shown that $\mathscr{L}$ is unitarily equivalent to an $\mathscr{L}(X, \leqq, m)$ where $X$ is a compact separable metric space, $\leqq$ is a standard partial ordering, and $m$ is a finite Borel measure. ([1], Theorem 1.3.1.) Hence, one approach to studying commutative lattices is to examine the relationships between the properties of the partially ordered measure space $(X, \leqq, m)$ and the properties of $\mathscr{L}(X, \leqq, m)$.

For this approach to be fruitful given a specific lattice $\mathscr{L}$ it would be desirable to obtain a reasonably concrete representation of $\mathscr{L}$. For example, Kadison and Singer have shown that if $\mathscr{L}$ is a multiplicity free chain, then $\mathscr{L}$ is unitarily equivalent to $\mathscr{L}([0,1]$, $\leqq, m)$ where $\leqq$ is the reverse of the usual ordering on $[0,1]$ and $m$ is a finite Borel measure with certain properties. In particular, if $\mathscr{L}^{\prime \prime}$ is nonatomic, then $m$ is Lebesgue measure. ([4], Chapter 3.)

In this section we generalize this result of Kadison-Singer to obtain that a multiplicity free finite width lattice $\mathscr{L}$ generated by $n$ chains is unitarily equivalent to $\mathscr{L}\left([0,1]^{n}, \leqq, m\right)$ where $\leqq$ is the product partial ordering on $[0,1]^{n}$ and $m$ is a finite Borel measure. (The product partial ordering on $[0,1]^{n}$ is such that $x \leqq y$ iff $x_{i} \leqq y_{i}$ for $i=1, \cdots, n$ where $x=\left(x_{1}, \cdots, x_{n}\right)$ and $y=\left(y_{1}, \cdots, y_{n}\right)$.) In the next section we examine the properties of the measure $m$.

Let $\mathscr{N}$ be a chain of projections on $\mathscr{H}$. Since $\mathscr{N}^{\prime \prime}$ is commutative we can imbed it in a m.a.s.a. Let $v$ be a unit cyclic vector for this m.a.s.a. Then $v$ is also a separating vector for the m.a.s.a. and hence for $\mathscr{N}^{\prime \prime}$, i.e., if $P v=0$ for $P \in \mathscr{N}^{\prime \prime}$, then $P=0$. Label $P \in \mathscr{N}$ by $P_{t}$ if $(P v, v)=t$. Note that $t \in[0,1]$ and that the labeling is well defined since $v$ is separating. Let $S_{\mathscr{r}}=\{t \in[0,1]$ : there exists a $\left.P_{t} \in \mathscr{N}\right\}$. We will call $S_{\mathscr{N}}$ the index set of $\mathscr{N}$ with respect to $v$. $S_{\mathscr{S}}$ contains 0 and 1 since $\mathscr{N}$ contains 0 and $I$. We also 
have that, for $s$ and $t$ in $S_{\mathscr{r}}$, if $s<t$, then $P_{s}<P_{t}$. By considering increasing and decreasing sequences in $S_{\mathscr{r}}$ and applying the chain properties of $\mathscr{N}$, we see that $S_{\mathscr{r}}$ is closed.

We will need the following lemma.

LEMMA 3.1. Let $\mathscr{C}$ be a chain of projections on $\mathscr{H}$. Then there exists a self-adjoint operator $A$ whose spectrum is contained in $[0,1]$ such that the projections in $\mathscr{C}$ are spectral projections of $A$ corresponding to the sets $[t, 1], 0 \leqq t \leqq 1$, and such that $A \in \mathscr{C}^{\prime \prime}$.

Proof. The construction of $A$ is a fairly standard process. We include an outline here since we refer to various parts of the construction throughout Theorem 3.3.

Define $\mathscr{D}$ as $\{I-E: E \in \mathscr{C}\}$ where $I$ is the identity projection. Choose a unit separating vector $v$ for $\mathscr{D}^{\prime \prime}$ as above. Let $S=S_{\mathscr{X}}$ be the index set of $\mathscr{D}$ with respect to $v$. (We introduce $\mathscr{D}$ so that our construction applies directly to our needs in Theorem 3.3 for projections corresponding to increasing Borel sets of $[0,1]$. The problem is that, relative to the normal ordering of $[0,1]$, the sets $[t, 1]$ (and not $[0, t]$ ) are increasing).

Let $x \in \mathscr{H}$. Define a function $f_{x x}$ on $S$ by $f_{x x}(t)=\left(D_{t} x, x\right)$. Extend $f_{x x}$ to $g_{x x}$ on $\boldsymbol{R}$ defined by

$$
g_{x x}(p)=\inf _{\substack{t \in S \\ p \leqq t}} f_{x x}(t) \text { and } g_{x x}(p)=f_{x x}(1) \text { if } p \geqq 1 .
$$

Note that $g_{x x}$ is bounded by $\|x\|^{2}$, is monotone increasing, and continuous on the left. By a standard measure theory result, there exists a measure $m_{x x}$ on the Borel sets of $\boldsymbol{R}$ defined by $m_{x x}\left(\left[t_{1}, t_{2}\right)\right)=$ $g_{x x}\left(t_{2}\right)-g_{x x}\left(t_{1}\right)$. By polarization we obtain a measure $m_{x y}$ for each $x$ and $y$ in $\mathscr{H}$ such that $\left|m_{x y}\right| \leqq\|x\|\|y\|, m_{x y}=\bar{m}_{y x}$, and $m_{x y}$ is linear in $x$ and conjugate linear in $y$.

By the properties of $m_{x y}$ we can define a map $P$ from the Borel sets of $\boldsymbol{R}$ into self-adjoint operators on $\mathscr{H}$ by $(P(M) x, y)=m_{x y}(M)$ where $M$ is a Borel subset of $\boldsymbol{R}$. Letting $t^{\prime}$ stand for $\inf \{s \in S: t \leqq s\}$, we have that for $t_{2} \geqq t_{1}$,

$$
\left(P\left(\left[t_{1}, t_{2}\right)\right) x, x\right)=g_{x x}\left(t_{2}\right)-g_{x x}\left(t_{1}\right)=f_{x x}\left(t_{2}^{\prime}\right)-f_{x x}\left(t_{1}^{\prime}\right)=\left(D_{t_{2}^{\prime}} x, x\right)-\left(D_{t_{1}^{\prime}} x, x\right) .
$$

This implies that $P\left(\left[t_{1}, t_{2}\right)\right)=D_{t_{2}^{\prime}}-D_{t_{1}^{\prime}}$ and hence that $P\left(\left[t_{1}, t_{2}\right)\right)$ is a projection (since $\mathscr{D}$ is a chain) and that $P\left(\left[t_{1}, t_{2}\right)\right) P\left(\left[s_{1}, s_{2}\right)\right)=P\left(\left[t_{1}, t_{2}\right) \cap\right.$ $\left.\left[s_{1}, s_{2}\right)\right)$. By a measure theoretical argument, we can conclude that $P(M)$ is a projection for every Borel subset $M$ of $\boldsymbol{R}$. Since the index set $S$ of $\mathscr{D}$ is closed, $R-S$ is a countable union of disjoint intervals $\left(l_{k}, r_{k}\right)$ where $l_{k}$ and $r_{k}$ are in $S$. Writing $\left(l_{k}, r_{k}\right)$ as the union of half closed intervals $\left[l_{k}+1 / n, r_{k}\right), n=1,2, \cdots$, we see from the 
definition of $m_{z z}, \quad z \in \mathscr{H}$, that $m_{z z}\left(\left(l_{k}, r_{k}\right)\right)=0$ and hence that $m_{x y}(\boldsymbol{R}-S)=0$. Hence we have that $P$ is a real spectral measure with compact support.

Define $A$ on $\mathscr{H}$ to be the operator $\int t d P(t)$, i.e., $(A x, y)=\int t d m_{x y}(t)$ for $x$ and $y$ in $\mathscr{H}$. From the facts that $m_{x y}=\bar{m}_{x y},\left|m_{x y}\right| \leqq\|x\|\|y\|$, and $m_{x y}\left(S^{c}\right)=0$ with $S \subset[0,1]$, we get that $A$ is self-adjoint (Hermitian), positive, and of norm less than or equal to 1 . Note that this implies that the spectrum of $A$ is contained in $[0,1]$.

Since $P$ is a real compact spectral measure such that $A$ is $\int t d P(t)$, it is the unique spectral measure associated with $A$. Hence the spectral projection of $A$ corresponding to the set $[t, 1]$ is $P([t, 1])$. We wish to show that $\{P([t, 1]): 0 \leqq t \leqq 1\}=\mathscr{C}$. Let $E \in \mathscr{C}$. Let $D=I-E$ and label $D$ by $D_{t_{0}}$ if $(D v, v)=t_{0}$. Note by definition, that $t_{0}$ is in $S$. Then,

$$
\begin{aligned}
\left(P\left(\left[t_{0}, 1+\varepsilon\right)\right) x, x\right) & =m_{x x}\left(\left[t_{0}, 1+\varepsilon\right)\right)=f_{x x}(1)-f_{x x}\left(t_{0}\right) \\
& =\left(D_{1} x, x\right)-\left(D_{t_{0}} x, x\right)=\left(\left(I-D_{t_{0}}\right) x, x\right)=(E x, x)
\end{aligned}
$$

for every $\varepsilon>0$ and every $x \in \mathscr{K}$. Hence, we have a $t_{0}$ in $[0,1]$ such that $P\left(\left[t_{0}, 1\right]\right)=E$. Now let $t \in[0,1]$. Let $t^{\prime}=\inf \{s \in S: t \leqq s\}$. We have that for all $\varepsilon>0$,

$$
\left(P\left(\left[t_{0}, 1+\varepsilon\right)\right) x, x\right)=f_{x x}(1)-f_{x x}\left(t^{\prime}\right)=\left(\left(I-D_{t^{\prime}}\right) x, x\right) .
$$

Hence $P([t, 1])=I-D_{t^{\prime}}$ an element of $\mathscr{C}$. If we also label the elements of $\mathscr{C}$ by $E=E_{r}$ if $(E v, v)=r$, then we can express the above as $P([t, 1])=E_{1-t^{\prime}}$ where $t^{\prime}=\inf \{s \in S: t \leqq s\}$.

We have left to show that $A \in \mathscr{C}^{\prime \prime}$. Let $\varepsilon>0$. Cover $[0,1]$ by disjoint half open intervals of length less than or equal to $\varepsilon$, i.e.,

$$
[0,1] \subset[0, \varepsilon) \cup[\varepsilon, 2 \varepsilon) \cup \cdots \cup[n \varepsilon,(n+1) \varepsilon)=M_{0} \cup \cdots \cup M_{n}
$$

where $1 /(n+1)<\varepsilon$. Choose $t_{i} \in M_{i}$. Let $T_{\varepsilon}=\sum_{i=1}^{n} t_{i} P\left(M_{i}\right)$. Then $T$ is a finite linear combination of elements in $\mathscr{D}$ and $\left\|A-T_{\varepsilon}\right\|<\varepsilon$. Hence $A$ is the norm limit of operators in $\mathscr{D}^{\prime \prime}$ and hence $A \in$ $\mathscr{D}^{\prime \prime}=\mathscr{C}^{\prime \prime}$.

We wish to observe the following before continuing.

Proposition 3.2. Let $\mathscr{N}$ be a chain of projections on $\mathscr{H}$ such that $\mathscr{N}^{\prime \prime}$ is nonatomic. Let $v$ be a unit separating vector for $\mathscr{N}^{\prime \prime}$. Then the index set of $\mathscr{N}$ with respect to $v$ is $[0,1]$.

Proof. The proof of this is essentially contained in KadisonSinger's paper ([4], Thm. 3.3.1 and Lemma 2.3.4). Label $P \in \mathscr{N}$ by 
$P_{r}$ if $(P v, v)=r$. Now let $t \in[0,1]$. The crux of the proof is showing that the difference between $\bigvee_{r<t} E_{r}$ and $\Lambda_{r>t} E_{r}$ is either zero, in which case both expressions equal the desired $E_{t}$, or it is a minimal projection in $\mathscr{N}^{\prime \prime}$, contradicting nonatomicity.

This implies for our construction in the lemma that $P([t, 1])=$ $E_{1-t}$ if $\mathscr{C}^{\prime \prime}$ is nonatomic. Our correspondence between spectral projections and $\mathscr{C}$ is thus more direct. We will first prove our representation theorem with the added hypothesis of nonatomicity on each chain and then indicate the quality of the changes if we drop this hypothesis.

TheOREM 3.3(1). Let $\mathscr{L}=\mathscr{C}_{1} \bigvee \cdots \bigvee \mathscr{C}_{n}$ be a finite width multiplicity free lattice where $\mathscr{C}_{i}^{\prime \prime}$ is nonatomic for each $i$. Then there exists a finite Borel measure $m$ on $[0,1]^{n}$ and a unitary operator $U: L^{2}\left([0,1]^{n}, m\right) \rightarrow \mathscr{H}$ such that $U \mathscr{L}\left([0,1]^{n}, \leqq, m\right) U^{-1}=\mathscr{L}$ where $\leqq$ is the product partial ordering on $[0,1]^{n}$.

Proof. Since $\mathscr{L}^{\prime \prime}$ is a m.a.s.a, there exists a unit cyclic vector for $\mathscr{L}^{\prime \prime}$ in $\mathscr{H}$. Call this vector $v$. We have that $v$ is also separating for $\mathscr{L}^{\prime \prime}$. Lavel $E \in \mathscr{C}_{i}$ by $E=E_{t}^{\imath}$ if $(E v, v)=t$. For each $\mathscr{C}_{i}$ construct $A_{i}$ as in Lemma 3.1 using $v$ in each construction. Let $P_{i}$ denote the unique spectral measure associated with $A_{i}$. We have that $A_{i}$ is a self-adjoint operator whose spectrum is in $[0,1], A_{i} \in \mathscr{C}_{i}^{\prime \prime}$, and $P_{i}([t, 1))=E_{1-t}^{i}$ for $0 \leqq t \leqq 1$.

Since $\mathscr{L}^{\prime \prime}$ is commutative and $\mathscr{C}_{i}^{\prime \prime} \subset \mathscr{L}^{\prime \prime}$ for each $i$, the $C^{*}$ algebra generated by $A_{1}, \cdots, A_{n}, I$ is commutative. Denote the algebra by $\mathfrak{A}$. Let $X^{\prime}$ be the maximal ideal space of $\mathfrak{A}$, i.e., the set of nonzero multiplicative linear functionals on $\mathfrak{A}$. By the association $\psi \rightarrow\left(\psi\left(A_{1}\right), \cdots, \psi\left(A_{n}\right)\right)$ where $\psi \in X^{\prime}, X^{\prime}$ becomes homeomorphic to a closed subset of the Cartesian product of the spectrums of $A_{1}, \cdots, A_{n}$ ([2], Theorem 11, §IX. 2). Let $X$ be this subset. We have that $X \subset[0,1]^{n}$.

By the Gelfand theory, we obtain an isometric isomorphic * representation $R: \mathscr{C}(X) \rightarrow \mathfrak{A}$ where $R\left(p\left(t_{1}, t_{2}, \cdots, t_{n}\right)\right)=p\left(A_{1}, \cdots, A_{n}\right)$ for $p$ a polynomial on $X$. For $x$ and $y$ in $\mathscr{H}$, define a linear functional $r_{x y}$ on $\mathscr{C}(X)$ by $r_{x y}(f)=(R(f) x, y)$. By the Riesz representation theorem, this corresponds to a unique finite Borel measure on $X$, call it $w_{x y}$, such that $(R(f) x, y)=\int f d w_{x y}$ for $f \in \mathscr{C}(X)$. Extend $w_{x y}$ to a measure on the Borel sets of $[0,1]^{n}$ by $w_{x y}(V)=w_{x y}(V \cap X)$ where $V$ is a Borel subset of $[0,1]^{n}$. We can extend $R$ to a * homomorphism from bounded Borel functions on $[0,1]^{n}$ into $\mathscr{L}(\mathscr{H})$ by $(R(f) x, y)=\int_{[0,]^{n}} f d w_{x y}$. (See 2, Theorem 1. $\S \mathrm{X}$. 2.)

Let $I=[0,1] . \quad$ (There should be no confusion with $I$ referring 
to the identity projection.) We now claim that $R\left(\chi_{I \times \cdots \times[t, 1] \times \cdots \times I}\right)=$ $E_{1-t}^{i}$ where $[t, 1]$ is the $i$ th position. This result is the basis of the rest of the proof.

We first make the following observation: Let $w$ be any Borel measure on $[0,1]^{n}$ and let $w^{i}$ be a measure on the Borel sets of $[0,1]$ such that $w^{i}(M)=w(I \times \cdots \times M \times \cdots \times I)$ where $M$ is in the $i$ th position. Let $f$ be a positive, bounded measurable function on $[0,1]^{n}$ such that $f(\bar{t})=g\left(t_{i}\right)$ where $\bar{t}=\left(t_{1}, \cdots, t_{n}\right)$ and $g$ is a bounded measurable function on $[0,1]$. We then have by the observation above that

$$
\int_{[0,1]^{n}} f(\bar{t}) d w(\bar{t})=\int_{[0,1]} g(t) d w^{i}(t) .
$$

Let $\bar{t}=\left(t_{1}, \cdots, t_{n}\right)$ be in $[0,1]^{n}$ and $q_{i}(\bar{t})=t_{i}$. Then

$$
\begin{aligned}
& A_{i}=R\left(q_{i}\right) \quad \text { and } \quad\left(A_{i} x, y\right)=\left(R\left(q_{i}\right) x, y\right)=\int_{[0,1]^{n}} q_{i}(\bar{t}) d w_{x y}(\bar{t}) \\
& =\int_{[0,1]} t d w_{x y}^{i}(t) \text {. }
\end{aligned}
$$

Define a projection valued function on the Borel sets of $\boldsymbol{R}$ by $Q_{i}(N)=R\left(\chi_{I \times \cdots \times N \cap I \times \cdots \times I}\right)$ where $N \cap I$ is in the $i$ th position. Using the properties of $R$, one can verify that $Q_{i}$ is a compact, real spectral measure and that $w_{x y}^{i}(M)=\left(Q_{i}(M) x, y\right)$ for $M$ a Borel subset of $[0,1]$. Hence we have that $A_{i}=\int t d Q_{i}(t)$. By the uniqueness of spectral measures associated with a given self-adjoint operator we must have that $Q_{i}=P_{i}$. We thus get, for $[t, 1]$ in the $i$ th position, that

$$
R\left(\chi_{I \times \cdots \times[t, 1] \times \cdots \times I}\right)=Q_{i}([t, 1])=P_{i}([t, 1])=E_{1-t}^{i} .
$$

We are now in a position to construct a measure $m$ and a unitary operator $U$ and show that they are what is needed to get the desired unitary equivalence. Let $v$ be the unit cyclic vector for $\mathscr{L}^{\prime \prime}$ chosen earlier. Define $m$ to be the Borel measure on $[0,1]$ given by $w_{v v}$. Note that $m(V)=\int \chi_{V} d w_{v v}=\left(R\left(\chi_{V}\right) v, v\right)$. We have the following equalities for $f$ a bounded Borel function on $[0,1]^{n}$ :

$$
\|R(f) v\|^{2}=(R(f) v, R(f) v)=(R(f \bar{f}) v, v)=\int|f|^{2} d w_{v v}=\int|f|^{2} d m .
$$

Let $L^{\infty}\left([0,1]^{n}, m\right)$ be the $m$-equivalence classes of bounded Borel functions on $[0,1]^{n}$. Define $U_{0}: L^{\infty}\left([0,1]^{n}, m\right) \rightarrow \mathscr{C}$ by $U_{0} f=R(f) v$. The above equalities show that $U_{0}$ is well defined and isometric in the $L^{2}\left([0,1]^{n}, m\right)$ norm. Since $L^{\infty}$ is dense in $L^{2}$, we can extend $U_{0}$ to a map $U$ from $L^{2}\left([0,1]^{n}, m\right)$ into $\mathscr{H}$. We claim that $U$ is unitary. 
To show that $U$ is unitary we need to show that the range of $U_{0}$ is dense in $\mathscr{H}$. Let $r(R)=\left\{R(f): f \in L^{\infty}\left([0,1]^{n}, m\right)\right\}$. We first claim that $r(R)$ is strongly dense in $\mathscr{L}^{\prime \prime}$, i.e., that $r(R)^{\prime \prime}=\mathscr{L}^{\prime \prime}$. By the result that $R\left(\chi_{I \times \cdots \times[t, 1] \times \cdots \times I}\right)=E_{1-t}^{i}$ where $[t, 1]$ is in the $i$ th position, we have that $\mathscr{C}_{i} \subset r(R)$ for each $i$. Now since $P \wedge Q=$ $P Q$ and $P \vee Q=P+Q-P Q$ for commutative projections, $\mathscr{L}=$ $\mathscr{C}_{1} \mathrm{~V} \cdots \vee \mathscr{C}_{n} \subset r(R)^{\prime \prime}$. Hence $\mathscr{L}^{\prime \prime} \subset r(R)^{\prime \prime}$. To get the inclusion the other way, let $T$ be in $r(R)^{\prime \prime}$. Since $\mathscr{C}_{i} \subset r(R)$ and $r(R)$ is commutative, $T$ commutes with each $\mathscr{C}_{i}$ and hence with $\mathscr{L}$. Since $T$ commutes with $\mathscr{L}$, it commutes with $\mathscr{L}^{\prime \prime}$. Since $\mathscr{L}^{\prime \prime}$ is a m.a.s.a, we have that $T$ is in $\mathscr{L}^{\prime \prime}$ which implies that $r(R)^{\prime \prime} \subset \mathscr{L}^{\prime \prime}$. Now, since $r(R)$ is strongly dense in $\mathscr{L}^{\prime \prime}$, for $T$ in $\mathscr{L}^{\prime \prime}$ there is a sequence $S_{n}$ of operators in $r(R)$ such that $S_{n} \rightarrow T$ strongly. Hence we get that $\left\{T v: T \in \mathscr{L}^{\prime \prime}\right\}$ is contained in the norm closure of $\{S v: S \in r(R)\}$. Since the norm closure of $\left\{T v: T \in \mathscr{L}^{\prime \prime}\right\}$ is $\mathscr{H}$ (because $v$ is cyclic for $\mathscr{L}^{\prime \prime}$ ), we conclude that the norm closure of $\{S v: S \in r(R)\}$ is $\mathscr{H}$, i.e., the norm closure of the range of $U_{0}$ is $\mathscr{L}$, our desired result.

We thus have a finite Borel measure $m$ and a unitary operator $U: L^{2}\left([0,1]^{n}, m\right) \rightarrow \mathscr{H}$. We wish to show that $U \mathscr{L}\left([0,1]^{n}, \leqq, m\right) U^{-1}=\mathscr{L}$ where $\leqq$ is the product ordering on $[0,1]^{n}$, i.e., $\bar{t} \leqq \bar{s}$ iff $t_{i} \leqq s_{i}$ for $i=1, \cdots, n$. We will accomplish this by showing that $U \cdot U^{-1}$ maps a generating set of $\mathscr{L}\left([0,1]^{n}, \leqq, m\right)$ onto a generating set for $\mathscr{L}$. (By a generating set for a lattice $\mathscr{L}$ we mean a subset $L$ of $\mathscr{L}$ such that $L$ is the strong closure of finite joins and meets of elements of $L$.)

We first make the following observation. For $f \in L^{\infty}\left([0,1]^{n}, m\right)$, let $L_{f}$ denote the operator on $L^{2}\left([0,1]^{n}, m\right)$ determined by multiplication by $f$. We claim that $U L_{f} U^{-1}=R(f)$. Let $g$ be in $L^{\infty}\left([0,1]^{n}, m\right)$ and let $v$ be our cyclic vector. We have

$$
U L_{f} g=U f g=R(f g) v=R(f) R(g) v=R(f) U g .
$$

The result follows for $g \in L^{2}$ since $L^{\infty}$ is dense in $L^{2}$.

Let $\bar{t}=\left(t_{1}, \cdots, t_{n}\right)$ and $\bar{s}=\left(s_{1}, \cdots, s_{n}\right)$ be in $[0,1]^{n}$. Let $F_{i, r}$ be be the Borel subset of $[0,1]^{n}$ given by $I \times \cdots \times[r, 1] \times \cdots \times I$ where $[r, 1]$ is in the $i$ th position. Note that $t_{i} \leqq s_{i}$ iff $\chi_{F_{i, r}}(\bar{t}) \leqq$ $\chi_{F_{i, r}}(\bar{s})$ for $r$ running over all rational numbers in $[0,1]$. We thus have for $\leqq$, the product ordering on $[0,1]^{n}$, that $\bar{t} \leqq \bar{s}$ iff $\chi_{F_{i, r}}(\bar{t}) \leqq$ $\chi_{F_{i, r}}(\bar{s})$ for $i=1, \cdots, n$ and all rational $r$ in $[0,1]^{n}$. Let $P_{i, r}$ be the projection on $L^{2}\left([0,1]^{n}, m\right)$ determined by multiplication by $\chi_{F_{i, r}}$. Since $\left\{F_{i, r}: i=1, \cdots, n, r\right.$ rational $\}$ is a countable set of Borel subsets of $[0,1]^{n}$, we have that $\left\{P_{i, r}: i=1, \cdots, n, r\right.$ rational $\}$ generates $\mathscr{L}\left([0,1]^{n}, \leqq, m\right) . \quad([1]$, Theorem 1.2.2.)

Let $L=\left\{U P_{i, r} U^{-1}: i=1, \cdots, n, r\right.$ rational $\}$. We wish to show that $L$ generates $\mathscr{L}$. By the observation above, 


$$
U P_{i, r} U^{-1}=R\left(\chi_{I \times \cdots \times[r, 1] \times \cdots \times I}\right)=E_{1-r}^{i} .
$$

Since $E_{1-r}^{i} \in \mathscr{C}_{i}$, we have that $L$ is contained in $\mathscr{L}$. Let $V_{i}=$ $\left\{E_{1-r}^{i} \in \mathscr{C}_{i}: r\right.$ is rational $\}$. If we can show that $V_{i}$ is strongly dense in $\mathscr{C}_{i}$, then the strongly closed lattice generated by $L$ will contain each $\mathscr{C}_{i}$ and hence $\mathscr{L}$. Let $E \in \mathscr{C}_{i}$. Let $t \in[0,1]$ such that $E=E_{t}^{i}$, i.e., $(E v, v)=t$ where $v$ is our unit cyclic vector. Let $\left\{t_{j}\right\}$ be a sequence of rational numbers converging to $t$. Let $E_{t_{j}}^{i}$ be the projection in $\mathscr{C}_{i}$ such that $\left(E_{t_{j}}^{i} v, v\right)=t_{j}$. Then

$$
\begin{aligned}
\left\|\left(E_{t_{j}}^{i}-E_{t}^{i}\right) v\right\|^{2} & =\left(\left(E_{t_{j}}^{i}-E_{t}^{i}\right) v,\left(E_{t_{j}}^{i}-E_{t}^{i}\right) v\right) \\
& =\left(\left(E_{t}^{i}-E_{t_{j}}^{i}\right) v,\left(E_{t}^{i}-E_{t_{j}}^{i}\right) v\right)=\left|t_{j}-t\right|
\end{aligned}
$$

since either $E_{t}^{i}-E_{t_{j}}^{i}$ or $E_{t_{j}}^{i}-E_{t}^{i}$ is a projection. We thus get that $E_{t_{j}}^{i} v$ converges to $E_{t}^{i} v$ as $j$ goes to $\infty$. Since $\mathscr{C}_{i}$ commutes with $\mathscr{L}^{\prime \prime}$ and $v$ is cyclic for $\mathscr{L}^{\prime \prime}$, we have that $\left\{E_{t_{j}}^{i}\right\}$ converges strongly to $E_{t}^{i}$. Letting $r_{j}=1-t_{j}$, we have that $E_{t}^{i}$ is the strong limit of the sequence $\left\{E_{1-r_{j}}^{i}\right\}$, i.e., that any projection in $\mathscr{C}_{i}$ is a strong limit of projections in $V_{i}$. We thus have our desired result that $L=$ $\left\{U P_{i, r} U^{-1}: i=1, \cdots, n, r\right.$ rational $\}$ generates $\mathscr{L}$.

Our proof is complete. Let $\leqq$ be the product ordering on $[0,1]^{n}$. We have constructed a finite Borel measure $m$ on $[0,1]^{n}$ and a unitary operator $U: L^{2}\left([0,1]^{n}, m\right) \rightarrow \mathscr{H}$ such that $U \cdot U^{-1}$ maps a generating set for $\mathscr{L}\left([0,1]^{n}, \leqq, m\right)$ onto a generating set for $\mathscr{L}$, i.e.,

$$
U \mathscr{L}\left([0,1]^{n}, \leqq m\right) U^{-1}=\mathscr{L} \text {. }
$$

THEOREM 3.3(2). Theorem 3.3(1) is valid without the hypothesis that each $\mathscr{C}_{i}^{\prime \prime}$ be nonatomic.

Proof. The changes in the proof are minimal. Let $\mathscr{D}_{i}=$ $\left\{I-E: E \in \mathscr{C}_{i}\right\}$. Let $v$ be our unit separating cyclic vector for $\mathscr{L}^{\prime \prime}$. Let $S_{i}$ be the index set of $\mathscr{D}_{i}$, i.e., $S_{i}=\left\{t \in[0,1]\right.$ : there exists $D \in \mathscr{D}_{i}$ such that $(D v, v)=t\}$. One basic change is that whenever we referred to the fact that $P_{i}([t, 1])=E_{1-t}^{i}$ before we would now have to say that $P_{i}([t, 1])=E_{1-t^{\prime}}^{i}$, where $t^{\prime}=\inf \left\{s \in S_{i}: t \leqq s\right\}$. The only other major change is in the final argument that $\left\{E_{1-r}^{i}: r\right.$ rational $\}$ is dense in $\mathscr{C}_{i}$. We would now have to argue that

$$
\left\{E_{1-r^{\prime}}^{i}: r^{\prime}=\inf \left\{s \in S_{i}: r \leqq s\right\} \text { where } r \text { is rational }\right\}
$$

is dense in $\mathscr{C}_{i}$. We omit the details.

4. Properties of the representation measure. In this section we will discuss some of the properties of the representation measure $m$ obtained in the construction in Theorem 3.3. We define the $i$ th 
marginal measure of $m$ as the Borel measure $m_{i}$ on $[0,1]$ defined by $m_{i}(M)=m(I \times \cdots \times M \times \cdots \times I)$ where $M$ is a Borel subset of $[0,1], I=[0,1]$, and $M$ is in the $i$ th position of the cross product.

CoROLlary 4.1. Let $\mathscr{L}=\mathscr{C}_{1} \mathrm{~V} \cdots \vee \mathscr{C}_{n}$ be a multiplicity free finite width lattice represented as $\mathscr{L}\left([0,1]^{n}, \leqq, m\right)$ as in Theorem 3.3. Let $S_{i}$ be the index set of $\mathscr{D}_{i}=\left\{I-E: E \in \mathscr{C}_{i}\right\}$. Express $[0,1]-S_{i}$ as $\mathrm{U}_{k} I_{k}$ where the $I_{k}$ are disjoint intervals $\left(l_{k}, r_{k}\right)$ with $l_{k}$ and $r_{k}$ in $S_{i}$. Then, for $M$ a Borel subset of $[0,1]$, we have

$$
m_{i}(M)=\mu\left(M \cap S_{i}\right)+\sum_{1_{k} \in M} \mu\left(I_{k}\right),
$$

where $\mu$ is Lebesgue measure on $[0,1]$. In particular, if $\mathscr{C}_{i}^{\prime \prime}$ is nonatomic, then $m_{i}$ is Lebesgue measure on $[0,1]$.

Proof. Denote the complement of $S_{i}$ in $[0,1]$ by $S_{1}^{c}$. Since $S_{i}$ is closed and contains 0 and 1 , it is possible to find the $I_{k}$. Let $l_{k}$ denote the left hand endpoint and $r_{k}$ the right hand endopoint of $I_{k}$. Note that $l_{k}$ and $r_{k}$ are in $S_{i}$.

We have that

$$
\begin{aligned}
m_{i}(M) & =m(I \times \cdots \times M \times \cdots \times I) \\
& =\left(R\left(\chi_{I \times \cdots \times M \times \cdots \times I}\right) v, v\right)=\left(P_{i}(M) v, v\right) .
\end{aligned}
$$

Recall that the measure $\left(P_{i}(\cdot) v, v\right)$ is the measure defined by a bounded monotonic function $h_{i}$ on $R$ where $h_{i}(t)=t^{\prime}=\inf \left\{s \in S_{i}\right.$ : $t \leqq s\}$. (See Lemma 3.1.) Note that $t^{\prime} \in S_{i}$.

It is sufficient to show the result for semi-closed intervals $\left[t_{1}, t_{2}\right)$ where $t_{1}$ and $t_{2}$ are in $[0,1]$ since these generate the Borel subsets of $[0,1]$. (Technically one should consider $\{[t, 1]: t \in[0,1]\}$ as part of the generating set but, since the arguments would be similar, we omit this detail.) Consider $\left[t_{1}, t_{2}\right)$. Let $K=\left\{k: l_{k} \in\left[t_{1}, t_{2}\right)\right\}$. We wish to show that $m_{i}\left(\left[t_{1}, t_{2}\right)\right)=\mu\left(\left[t_{1}, t_{2}\right) \cap S_{i}\right)+\sum_{k \in K} \mu\left(I_{k}\right)$.

For any $t_{1}, t_{2}$ in $[0,1]$, we have that $\left(\bigcup_{k \in K} I_{k}\right) \cup\left(\left[t_{1}, t_{2}\right) \cap S_{i}\right)=$ $\left[t_{1}^{\prime}, t_{2}^{\prime}\right)$. Since the union on the left is disjoint, we have

$$
\begin{gathered}
\mu\left(\left[t_{1}, t_{2}\right) \cap S_{i}\right)+\sum_{k \in K} \mu\left(I_{k}\right)=\mu\left(\left[t_{1}^{\prime}, t_{2}^{\prime}\right)\right)=t_{2}^{\prime}-t_{1}^{\prime} \\
=h_{i}\left(t_{2}\right)-h_{i}\left(t_{1}\right)=m_{i}\left(\left[t_{1}, t_{2}\right)\right) .
\end{gathered}
$$

In particular, if $\mathscr{C}_{i}^{\prime \prime}$ is nonatomic, then $S_{i}=[0,1]$ (see Prop. 3.2) and we have $m_{i}(M)=\mu(M)$ for $M$ a Borel subset of $[0,1]$ (and where $\mu$ is Lebesgue measure).

Even though the marginal measures of a measure on a product space may be quite nice, the measure itself can vary in structure considerably. (For example, consider $[0,1]^{2}$ and the following 
measures: $\mu \times \mu$, product Lebesgue measure, and $d$, the disgonal measure where $d(V)=\mu\{x \in[0,1]:(x, x) \in V\}$. Both measures have marginal measures equal to Lebesgue measure.)

Hence we investigate the structure of $m$ further. We introduce now the notion of independence of the chains in a finite width lattice. This will have useful consequences for the representation measure.

Let $\mathscr{L}=\mathscr{C}_{1} \mathrm{~V} \cdots \vee \mathscr{C}_{n}$ where each $\mathscr{C}_{i}$ is a chain. We say $\mathscr{C}_{1}, \cdots, \mathscr{C}_{n}$ are independent if given $E_{i}, F_{i} \in \mathscr{C}_{i}$ we have that

$$
E_{1} \wedge \cdots \wedge E_{n} \leqq F_{1} \vee \cdots \vee F_{n} \text { implies } E_{j} \leqq F_{j} \text { for some } j \text {. }
$$

We first wish to comment that a lattice of the form $\mathscr{C} \vee \mathscr{C}^{\perp}$ (where $\mathscr{C}^{\perp}=\{I-E: E \in \mathscr{C}\}$ and $\mathscr{C} \neq\{0, I\}$ ) does not satisfy this condition. (Recall from the introduction that a multiplicity free lattice $\mathscr{L}=\mathscr{C} \bigvee \mathscr{C}^{\perp}$ where $\mathscr{C}^{\prime \prime}$ is nonatomic cannot be left invariant by a compact operator.) Let $E_{1} \in \mathscr{C}, E_{1} \neq 0$ or $I$ and choose $F_{1}=0$. Choose $E_{2}=I-E_{1} \in \mathscr{C}^{\perp}$ and $F_{2}=0$. Then $E_{1} \wedge E_{2}=0=$ $F_{1} \vee F_{2}$ but $E_{1} \not \equiv F_{1}$ and $E_{2} \not F_{2}$.

In the corollary below we use the following equivalent characterization of independence since we are dealing with commutative lattices: Choose $E_{i}, F_{i} \in \mathscr{C}_{i}$ and let $\mathscr{C}_{i}=E_{i} F_{i}^{+}=E_{i}\left(I-F_{i}\right) . \quad \mathscr{C}_{1}, \cdots, \mathscr{C}_{n}$ are independent iff $C_{1} C_{2} \cdots C_{n}=0$ implies that $C_{j}=0$ for some $j$. (The proof is straightforwad since, for commutative projections, $\left(F_{1} \vee \cdots \vee F_{n}\right)^{\perp}=F_{1}^{\perp} F_{2}^{\perp} \cdots F_{n}^{\perp}$ and $E \leqq F$ iff $E F^{\perp}=0$.)

CoROllary 4.2. Let $\mathscr{L}=\mathscr{C}_{1} \bigvee \cdots \bigvee \mathscr{C}_{n}$ be a multiplicity free finite width lattice represented as $\mathscr{L}\left([0,1]^{n}, \leqq, m\right)$ as in Theorem 3.3. Assume $\mathscr{C}_{1}, \cdots, \mathscr{C}_{n}$ are independent. Then, for $r_{i}, s_{i} \in[0,1]$, $m\left(\left[r_{1}, s_{1}\right) \times \cdots \times\left[r_{n}, s_{n}\right)\right)=0$ implies that $m_{j}\left(\left[r_{j}, s_{j}\right)\right)=0$ for some $j$ where $m_{j}$ is the $j$ th marginal measure of $m$.

Proof. Recall that $m(V)=\left(R\left(\chi_{V}\right) v, v\right)$ for $V$ a Borel set $[0,1]^{n}$. Assume $m\left(\left[r_{1}, s_{1}\right) \times \cdots \times\left[r_{n}, s_{n}\right)\right)=0$ for $r_{i}, s_{i} \in[0,1]$.

Let $E_{i}=R\left(\chi_{I \times \cdots \times\left[r_{i}, 1\right] \times \cdots \times I}\right)$ and $F_{i}=R\left(\chi_{I \times \cdots \times\left[s_{i}, 1\right] \times \cdots \times I}\right)$ where $\left[s_{i}, 1\right]$ and $\left[r_{i}, 1\right]$ are in the $i$ th position of the cross products. We have that $E_{i}$ and $F_{i}$ are in $\mathscr{C}_{i}$. Then $C_{i}=E_{i} F_{i}^{\llcorner}=R\left(\chi_{I \times \cdots \times\left[r_{i}, s_{i}\right) \times \cdots \times I}\right)$ since $R$ is a homomorphism and $F_{i}^{\perp}=I-F_{i}=R\left(\chi_{I \times \cdots \times\left[0, s_{i}\right) \times \cdots I}\right)$. Let $V_{i}=$ $I \times \cdots \times\left[r_{i}, s_{i}\right) \times \cdots \times I$. Now,

$$
C_{1} \cdots C_{n}=\prod_{i=1}^{n} R\left(\chi_{V_{i}}\right)=R\left(\chi_{\Gamma_{i} V_{i}}\right)=R\left(\chi_{\left[r_{1}, s_{1}\right) \times \cdots \times\left[r_{n}, s_{n}\right]}\right)=0
$$

since $m\left(\left[r_{1}, s_{1}\right) \times \cdots \times\left[r_{n}, s_{n}\right)\right)=0$. Since $C_{1}, \cdots, C_{n}$ are independent, we have that $C_{j}=0$ for some $j$, i.e., for some $j, 0=C_{j}=$ 
$R\left(\chi_{I \times \cdots \times\left[r_{j}, s_{j}\right) \times I}\right)$. This in turn implies that $0=m\left(I \times \cdots \times\left[r_{j}, s_{j}\right) \times\right.$ $\cdots \times I)=m_{j}\left(\left[r_{j}, s_{j}\right)\right)$, our desired result. Note that if each $C_{i}^{\prime \prime}$ is nonatomic (which implies that each marginal measure of $m$ is Lebesgue measure), then $\mathrm{m}(W)>0$ for every open subset $W$ of $[0,1]^{n}$.

5. The desired Hilbert-Schmidt operator. Let $\mathscr{L}=$ $\mathscr{C}_{1} \mathrm{~V} \cdots \bigvee \mathscr{C}_{n}$ be a multiplicity free finite width lattice where $\mathscr{C}_{1}, \cdots, \mathscr{C}_{n}$ are independent. In this section we show that there exists a Hilbert-Schmidt operator on $\mathscr{H}$ that will leave $\mathscr{L}$ invariant.

We need the following lemma due to Arveson ([1], Prop. 1.6.0). For completeness, we include that part of the proof needed for Theorem 5.3.

Recall that a Hilbert-Schmidt operator $K$ on $L^{2}(X, m)$ can be expressed as an integral operator with kernel $k$ in $L^{2}(X \times X, m \times m)$, i.e., $K f(x)=\int k(x, t) f(t) d m(t)$. Recall also that the graph $G$ of the partial ordered set $(X, \leqq)$ is $\{(x, y) \in X \times X: y \leqq x\}$.

Lemma 5.1. Let $(X, \leqq, m)$ be a standard partially ordered measure space where $X$ is a separable metric space and $m$ is a $\sigma$-finite Borel measure. Let $T_{k}$ be a Hilbert-Schmidt operator on $L^{2}(X, m)$ with kernel $k$. Then $T_{k}$ leaves $\mathscr{L}(X, \leqq, m)$ invariant iff $k$ lives a.e. $(m \times m)$ on $G$, the graph of $(X, \leqq)$.

Proof. We prove only the if part since it is all we need for our purposes. Let $k$ live on $G$ a.e. $(m \times m)$. Let $P_{E}$ be the projection in $\mathscr{L}(X, \leqq, m)$ given by multiplication by $\chi_{E}$, where $E$ is an increasing set. We wish to show that $T_{k}$ leaves $P_{E}$ invariant, i.e., that $\left(T_{k} f, g\right)=0$ for $f \in P_{E} L^{2}(X, m)$ and $g \in\left(I-P_{E}\right) L^{2}(X, m)$. We have that $f$ lives on $E$ a.e. $(m)$ and $g$ lives on $X-E$ a.e. $(m)$. Now $\left\langle T_{k} f, g\right)=\int k(x, y) f(y) \overline{g(x)} d m(y) d m(x)$. The function $f(y) \bar{g}(x)$ lives a.e. $(m \times m)$ on $(X-E) \times E$ and $k(x, y)$ lives a.e. on $G$. Since $E$ is increasing, $(X-E) \times E$ is disjoint from $G$. Hence we have the desired result that $\left(T_{k} f, g\right)=0$.

THEOREM 5.3. Let $\mathscr{L}=\mathscr{C}_{1} \mathrm{~V} \cdots \vee \mathscr{C}_{n}$ be a finite width multiplicity free lattice where $\mathscr{C}_{1}, \cdots, \mathscr{C}_{n}$ are independent. Then there exists a Hilbert-Schmidt operator on $\mathscr{C}$ leaving $\mathscr{L}$ invariant.

Proof. We can assume without loss of generality that none of the $\mathscr{C}_{i}=\{0, I\}$. Represent $\mathscr{L}$ as $\mathscr{L}\left([0,1]^{n}, \leqq, m\right)$ as in Theorem 3.3. Let $U$ be the unitary operator from $L^{2}\left([0,1]^{n}, m\right)$ onto $\mathscr{X}$ that implements the unitary equivalence of these lattices. Let $m_{i}$ be the 
$i$ th marginal measure of $m$. Note that $G=\left\{(x, y) \in[0,1]^{n} \times[0,1]^{n}\right.$ : $y_{i} \leqq x_{i}$ for $i=1, \cdots, n$ where $x=\left(x_{1}, \cdots, x_{n}\right)$ and $\left.y=\left(y_{1}, \cdots, y_{n}\right)\right\}$.

We will be able to find intervals $\left[t_{i}^{1}, s_{i}^{1}\right]$ and $\left[t_{i}^{2}, s_{i}^{2}\right]$ for $i=1, \cdots, n$ with $s_{i}^{2} \leqq t_{i}^{1}$ such that neither $m_{i}\left(\left[t_{i}^{1}, s_{i}^{1}\right]\right)$ nor $m_{i}\left(\left[t_{i}^{2}, s_{i}^{2}\right]\right)$ are zero. Given such intervals we have that

$$
V=\prod_{i=1}^{n}\left[t_{i}^{1}, s_{i}^{1}\right] \times \prod_{i=1}^{n}\left[t_{i}^{2}, s_{i}^{2}\right] \subset G .
$$

By the independence condition we have that $m \times m(V)>0$ (see Corollary 4.2). Hence by letting $k(x, y)=\chi_{V}(x, y)$, we get a HilbertSchmidt operator $T_{k}$ with kernel $k$ living on $G$ and hence $T_{k}$ leaves $\mathscr{L}\left([0,1]^{n}, \leqq, m\right)$ invariant. Thus $U T_{k} U^{-1}$ is a Hilbert-Schmidt operator on $\mathscr{H}$ which leaves $\mathscr{L}$ invariant.

We now show that such intervals $\left[t_{i}^{1}, s_{i}^{1}\right]$ and $\left[t_{i}^{2}, s_{i}^{2}\right]$ exist for each $i$. Choose $i=1, \cdots, n$. Recall that if $S_{i}$ is the index set of $\mathscr{D}_{i}=$ $\left\{I-E: E \in \mathscr{C}_{i}\right\}$, then the $i$ th marginal measure $m_{i}$ satisfies $m_{i}(M)=$ $\mu\left(M \cap S_{i}\right)+\sum_{l_{k} \in M} \mu\left(I_{k}\right)$ where $M$ is a Borel subset of $[0,1], \mu$ is Lebesgue measure, and $I_{k}=\left(l_{k}, r_{k}\right)$ where the $I_{k}$ are disjoint intervals contained in $[0,1]-S_{i}$ and $l_{k}$ and $r_{k}$ are in $S_{i}$. (Corollary 4.1.) Suppose first that $[0,1]-S_{i}$ contains at least two disjoint nonempty intervals, say $\left(l_{1}, r_{1}\right)$ and $\left(l_{2}, r_{2}\right)$. Since they are disjoint with endpoints in $S_{i}$ we can assume that $r_{1}<l_{1}$. We have that, for $j=1,2$,

$$
m_{i}\left(\left[l_{j}, r_{j}\right]\right)=\mu\left(\left[l_{j}, r_{j}\right] \cap S_{i}\right)+\sum_{l_{k} \in\left[l_{j}, r_{j}\right]} \mu\left(I_{k}\right) \geqq \mu\left(\left[l_{j}, r_{j}\right]\right)>0 .
$$

Hence we can choose $\left[t_{i}^{1}, s_{i}^{1}\right]=\left[l_{1}, r_{1}\right]$ and $\left[t_{i}^{2}, s_{i}^{2}\right]=\left[l_{2}, r_{2}\right]$.

Now suppose $[0,1]-S_{i}$ is the empty set or the interval $(l, r)$ for some $l$ and $r$ in $S_{i}$. Since $\mathscr{C}_{i} \neq\{0, I\}$ (and hence $S_{i}$ is larger than $\{0,1\})$, this implies that $S_{i}$ properly contains a closed interval $[a, b]$. The marginal measure $m_{i}$ restricted to $[a, b]$ is Lebesgue measure restricted to $[a, b]$. Let $a<c<b$. Thus we can choose $\left[t_{j}^{1}, s_{i}^{1}\right]=[c, b]$ and $\left[t_{i}^{2}, s_{i}^{2}\right]=[a, c]$.

The proof is complete.

\section{REFERENCES}

1. W. Arveson, Operator algebras and invariant subspaces, Ann. of Math., 100 (1974), 433-532.

2. N. Dunford and J. Schwartz, Linear Operators, Part II, Interscience Publishers, New York-London, 1967.

3. P. R. Halmos, Introduction to Hibert Space, Chelsea Publishing Company, New York, N.Y., 1957.

4. R. Kadison and I. M. Singer, Triangular operator algebras, Amer. J. Math., 82 (1960), 227-259. 
5. J. R. Ringrose, On some algebras of operators, Proc. London Math. Soc., (3), 15 (1965), 61-83.

Received January 21, 1978 and in revised form July 14, 1978.

DALHOUSIE UNIVERSITY

HALIFAX

Nova Scotia, Canada 



\section{PACIFIC JOURNAL OF MATHEMATICS}

\section{EDITORS}

DONALD BABBITT (Managing Editor)

University of California

Los Angeles, CA 90024

HUGo RossI

University of Utah

Salt Lake City, UT 84112

C. C. MOORE and ANDREW OGG

University of California

Berkeley, CA 94720
J. DugundJI

Department of Mathematics

University of Southern California

Los Angeles, CA 90007

R. FinN and J. Milgram

Stanford University

Stanford, CA 94305

ASSOCIATE EDITORS
E. F. BECKENBACH
B. H. NeUmanN
F. WOLF
K. YoSHIDA

\section{SUPPORTING INSTITUTIONS}

UNIVERSITY OF BRITISH COLUMBIA

CALIFORNIA INSTITUTE OF TECHNOLOGY

UNIVERSITY OF CALIFORNIA

MONTANA STATE UNIVERSITY

UNIVERSITY OF NEVADA, RENO

NEW MEXICO STATE UNIVERSITY

OREGON STATE UNIVERSITY

UNIVERSITY OF OREGON
UNIVERSITY OF SOUTHERN CALIFORNIA

STANFORD UNIVERSITY

UNIVERSITY OF HAWAII

UNIVERSITY OF TOKYO

UNIVERSITY OF UTAH

WASHINGTON STATE UNIVERSITY

UNIVERSITY OF WASHINGTON

The Supporting Institutions listed above contribute to the cost of publication of this Journal, but they are not owners or publishers and have no responsibility for its content or policies.

Mathematical papers intended for publication in the Pacific Journal of Mathematics should be in typed form or offset-reproduced, (not dittoed), double spaced with large margins. Please do not use built up fractions in the text of the manuscript. However, you may use them in the displayed equations. Underline Greek letters in red, German in green, and script in blue. The first paragraph or two must be capable of being used separately as a synopsis of the entire paper. Please propose a heading for the odd numbered pages of less than 35 characters. Manuscripts, in triplicate, may be sent to any one of the editors. Please classify according to the scheme of Math. Reviews, Index to Vol. 39. Supply name and address of author to whom proofs should be sent. All other communications should be addressed to the managing editor, or Elaine Barth, University of California, Los Angeles, California, 90024.

50 reprints to each author are provided free for each article, only if page charges have been substantially paid. Additional copies may be obtained at cost in multiples of 50 .

The Pacific Journal of Mathematics is issued monthly as of January 1966. Regular subscription rate: $\$ 84.00$ a year (6 Vols., 12 issues). Special rato: $\$ 42.00$ a year to individual members of supporting institutions.

Subscriptions, orders for numbers issued in the last three calendar years, and changes of address shoud be sent to Pacific Journal of Mathematics, P.O. Box 969, Carmel Valley, CA 93924, U.S.A Old back numbers obtainable from Kraus Periodicals Co., Route 100, Millwood, NY 10546.

PUBLISHED BY PACIFIC JOURNAL OF MATHEMATICS, A NON-PROFIT CORPORATION

Printed at Kokusai Bunken Insatsusha (International Academic Printing Co., Ltd.). 8-8, 3-chome, Takadanobaba, Shinjuku-ku, Tokyo 160, Japan.

Copyright (C) 1980 by Pacific Jounal of Mathematics Manufactured and first issued in Japan 


\section{Pacific Journal of Mathematics \\ Vol. 89, No. $2 \quad$ June, 1980}

Frank Hayne Beatrous, Jr. and R. Michael Range, On holomorphic

approximation in weakly pseudoconvex domains................. 249

Lawrence Victor Berman, Quadratic forms and power series fields ...... 257

John Bligh Conway and Wacław Szymański, Singly generated antisymmetric operator algebras ....................... 269

Patrick C. Endicott and J. Wolfgang Smith, A homology spectral sequence for submersions . . . .................................

Sushil Jajodia, Homotopy classification of lens spaces for one-relator groups with torsion ................................ 301

Herbert Meyer Kamowitz, Compact endomorphisms of Banach

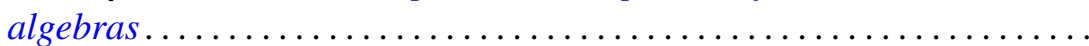

Keith Milo Kendig, Moiré phenomena in algebraic geometry: polynomial

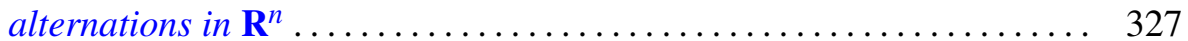

Cecelia Laurie, Invariant subspace lattices and compact operators....... 351

Ronald Leslie Lipsman, Restrictions of principal series to a real form . . . . . 367

Douglas C. McMahon and Louis Jack Nachman, An intrinsic

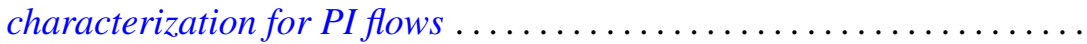

Norman R. Reilly, Modular sublattices of the lattice of varieties of inverse semigroups .................................... 405

Jeffrey Arthur Rosoff, Effective divisor classes and blowings-up of $\mathbf{P}^{2}$ 419

Zalman Rubinstein, Solution of the middle coefficient problem for certain

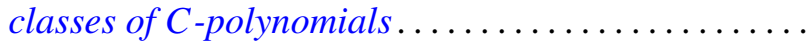

Alladi Sitaram, An analogue of the Wiener-Tauberian theorem for spherical transforms on semisimple Lie groups ................

Hal Leslie Smith, A note on disconjugacy for second order systems ...

J. Wolfgang Smith, Fiber homology and orientability of maps ...

Audrey Anne Terras, Integral formulas and integral tests for series of positive matrices. 